\title{
Towards the Kantorovich dual solution for strictly correlated electrons in atoms and molecules
}

\author{
Christian B. Mendl ${ }^{1}$ and Lin $\operatorname{Lin}^{2}$ \\ ${ }^{1}$ Mathematics Department, Technische Universität München, \\ Boltzmannstraße 3, 85748 Garching bei München, Germany \\ ${ }^{2}$ Computational Research Division, Lawrence Berkeley National Laboratory, Berkeley, California 94720, USA
}

\begin{abstract}
The many-body Coulomb repulsive energy of strictly correlated electrons provides direct information of the exact Hohenberg-Kohn exchange-correlation functional in the strong interaction limit. Until now the treatment of strictly correlated electrons is based on the calculation of co-motion functions with the help of semi-analytic formulations. This procedure is system specific and has been limited to spherically symmetric atoms and strictly $1 \mathrm{D}$ systems. We develop a nested optimization method which solves the Kantorovich dual problem directly, and thus facilitates a general treatment of strictly correlated electrons for systems including atoms and small molecules.
\end{abstract}

PACS numbers: 31.15.E-,71.27.+a

\section{INTRODUCTION}

Kohn-Sham density functional theory (KSDFT) is the most widely used electronic structure theory for systems with many electrons, from gas phase molecules to condensed matter systems in particular ${ }^{1,2}$. It is in principle an exact theory, and is able to yield the exact ground state energy and density using a fictitious system of non-interacting electrons. The key component of KSDFT is the exchange-correlation functional. Tremendous progress has been made in the past three decades for constructing approximate exchange-correlation functionals based on the known information from the uniform electron $\operatorname{gas}^{3-6}$. However, such approximate exchangecorrelation functionals are known to fail for strongly correlated systems, such as the chromium dimer ${ }^{7}$ or MottHubbard insulators ${ }^{8,9}$. Several recent studies indicate that the construction of exchange-correlation functionals for general strongly correlated systems can be extremely difficult ${ }^{9-11}$.

Recently, the behavior of the exchange-correlation functional has been revealed in the limit of strictly correlated electrons $(\mathrm{SCE})^{12-15}$. The many-body Coulomb repulsive energy of SCE determines the exact exchangecorrelation functional in the strong interaction limit, without artificially breaking any symmetry of the system or introducing any tunable parameters. The information provided by the SCE limit is complementary to that provided by the Kohn-Sham non-interacting kinetic energy. For given electron density profile, the SCE limit is described by minimizing the many-body Coulomb interaction energy with respect to all wavefunctions in a $3 N$ dimensional space, where $N$ is the number of electrons in the system, under the additional constraint that the wavefunction is consistent with the electron density $^{12}$. Mathematically, this daunting minimization task is an optimal transport problem with Coulomb cost function ${ }^{13,15,16}$. The optimal transport theory finds the optimal way for transferring masses from one position to another. The theory dates back to Monge in 1781, and was significantly generalized by Kantorovich in $1942^{17,18}$. We refer the readers to Ref. 19 for more detailed information. From physical intuition, the optimal transport problem with Coulomb cost function can be simplified by introducing $N$ co-motion functions $f_{i}: \mathbb{R}^{3} \rightarrow \mathbb{R}^{312}$. These co-motion functions characterize the relative positions of all the electrons with respect to one given electron in the SCE limit. To the extent of our knowledge, in practice the co-motion functions can only be determined for one dimensional systems ${ }^{14}$ and spherically symmetric atoms ${ }^{12,13,15}$, with the help of semianalytic methods. Little is known about the shape or even the existence of the co-motion functions for general systems including small molecules. On the other hand, the optimal transport problem with Coulomb cost function can be equivalently solved by its dual formulation, called the Kantorovich dual problem ${ }^{13,17-20}$. The main advantage of the Kantorovich dual problem is its potential applicability to general systems, ranging from atoms and molecules to condensed matter systems. However, the Kantorovich dual problem is formulated as a maximization problem with an infinite number of constraints, which is impossible to be implemented directly. These limitations severely restrict the applicability of the SCE limit to systems of practical interest. In this paper, we develop a novel method that solves the Kantorovich dual problem directly. We overcome the difficulty of infinite number of constraints via a nested optimization approach. Our method provides a more general treatment of the exchange-correlation functional in the SCE limit for atoms and small molecules.

The rest of the paper is organized as follows: in Section II, we briefly review the SCE limit, the optimal transport formulation and the Kantorovich dual problem, and present the nested optimization method for solving the Kantorovich dual problem directly. In Section III, we establish the applicability and accuracy of this method for the 3D beryllium atom and a model quantum wire system in 1D, for which accurate results can be obtained semi-analytically using the co-motion formulation. Next, 
we demonstrate the applicability of our method to a model trimer with various number of electrons in 3D, for which the SCE limit cannot be calculated by existing techniques. The conclusion and future work is given in Section IV.

\section{THEORY}

According to the Hohenberg-Kohn theorem ${ }^{1}$, the ground state energy of a system can be obtained by minimizing the following functional with respect to the electron density $\rho(\mathbf{r})$ :

$$
E[\rho]=F[\rho]+\int v_{\text {ext }}(\mathbf{r}) \rho(\mathbf{r}) \mathrm{d} \mathbf{r} .
$$

Here $v_{\text {ext }}(\mathbf{r})$ is the external potential, and $F[\rho]$ is the internal energy functional, which is a universal functional of the electron density and consists of the kinetic energy and the Coulomb repulsive energy between the electrons. Formally $F[\rho]$ is defined by minimizing over all the antisymmetric wavefunctions $\Psi$ which are consistent with $\rho(\mathbf{r})$ as

$$
F[\rho]=\min _{\Psi \rightarrow \rho}\left\langle\Psi\left|\hat{T}+\hat{V}_{e e}\right| \Psi\right\rangle .
$$

Here $\hat{T}=-\sum_{i=1}^{N} \frac{1}{2} \Delta_{i}$ is the kinetic energy operator and $\hat{V}_{e e}=\sum_{i=1}^{N} \sum_{j>i}^{N}\left|\mathbf{r}_{i}-\mathbf{r}_{j}\right|^{-1}$ is the Coulomb repulsive energy operator. $\Delta_{i}$ is the Laplacian operator on the $i$ th electron. The strong interaction limit considers the situation when the Coulomb repulsive energy dominates over the kinetic energy, in which case the internal energy functional can be approximated as ${ }^{14}$

$$
\begin{aligned}
F[\rho] & \approx \min _{\Psi \rightarrow \rho}\langle\Psi|\hat{T}| \Psi\rangle+\min _{\Psi \rightarrow \rho}\left\langle\Psi\left|\hat{V}_{e e}\right| \Psi\right\rangle \\
& \equiv T_{s}[\rho]+V_{e e}^{\mathrm{SCE}}[\rho] .
\end{aligned}
$$

The first term $T_{s}[\rho]$ is the Kohn-Sham (KS) kinetic energy functional corresponding to a non-interacting independent particle system ${ }^{2}$. The second term $V_{e e}^{\mathrm{SCE}}[\rho]$ is the minimal Coulomb repulsive energy among all wavefunctions which are consistent with $\rho(\mathbf{r})$, and the corresponding minimizer characterizes the state of "strictly correlated electrons" (SCE). In terms of KSDFT, $V_{e e}^{\mathrm{SCE}}[\rho]$ is the sum of the Hartree energy and the exchange correlation energy, and the exchangecorrelation functional in the SCE limit can be recovered by

$$
E_{\mathrm{xc}}^{\mathrm{SCE}}[\rho]=V_{e e}^{\mathrm{SCE}}[\rho]-\frac{1}{2} \iint \frac{\rho(\mathbf{r}) \rho\left(\mathbf{r}^{\prime}\right)}{\left|\mathbf{r}-\mathbf{r}^{\prime}\right|} \mathrm{d} \mathbf{r} \mathrm{d} \mathbf{r}^{\prime} .
$$

Eq. (3) allows for treating the kinetic energy functional and the Coulomb repulsive energy functional on the same footing but with different numerical techniques. The minimization of the kinetic energy functional $T_{s}[\rho]$ gives rise to a energy minimization problem or a nonlinear eigenvalue problem known as the Kohn-Sham equations. Their efficient treatment has been extensively explored in the past few decades. The minimization of the SCE functional $V_{e e}^{\mathrm{SCE}}[\rho]$ gives rise to an optimal transport problem, for which numerical methods are still very sparse. From the mathematical point of view, the results for the optimal transport problem are primarily concerned with quadratic cost functions. Rigorous treatment of the Coulomb cost function only appeared recently ${ }^{15}$ for $N=2$, and the formal description for general $N$ as well as results concerning the dual formulation have been introduced in Ref. 13. However, a proper mathematical generalization concerning the existence and uniqueness of the co-motion functions has not been achieved yet.

Formally, the optimal transport problem is solved by minimizing over all $3 N$-dimensional wavefunctions that are consistent with the given electron density $\rho(\mathbf{r})$. Following physical intuition ${ }^{12}$, the optimal transport problem can be solved by finding $N$ co-motion functions $\left\{\mathbf{f}_{1}(\mathbf{r}), \mathbf{f}_{2}(\mathbf{r}), \ldots, \mathbf{f}_{N}(\mathbf{r})\right\}, \mathbf{f}_{i}: \mathbb{R}^{3} \rightarrow \mathbb{R}^{3}$. Each $\mathbf{f}_{i}(\mathbf{r})$ represents the optimal position of the $i$-th electron given the position of the first electron at position $\mathbf{r}$, with the natural definition that $\mathbf{f}_{1}(\mathbf{r})=\mathbf{r}$. Since the electrons are indistinguishable and distributed according to the same density $\rho(\mathbf{r})$, the co-motion functions should satisfy the mass conservation constraint that $\rho\left(\mathbf{f}_{i}(\mathbf{r})\right) \mathrm{d} \mathbf{f}_{i}(\mathbf{r})=\rho(\mathbf{r}) \mathrm{d} \mathbf{r}$. Then $V_{e e}^{\mathrm{SCE}}[\rho]$ is given in terms of the co-motion functions by ${ }^{12,13}$

$$
V_{e e}^{\mathrm{SCE}}[\rho]=\frac{1}{N} \int \rho(\mathbf{r}) \sum_{i=1}^{N} \sum_{j>i}^{N} \frac{1}{\left|\mathbf{f}_{i}(\mathbf{r})-\mathbf{f}_{j}(\mathbf{r})\right|} \mathrm{d} \mathbf{r} .
$$

The co-motion functions are implicit functionals of the electron density, and can be obtained via semi-analytic formulations for spherical symmetric atoms ${ }^{12,15}$ and strictly $1 \mathrm{D}$ systems ${ }^{14}$. However, these semi-analytic formulations are system specific, and the co-motion functions cannot be obtained in practice even for general systems as simple as a dimer in 3D.

As an alternative to the co-motion framework, the Kantorovich dual formulation of the optimal transport problem ${ }^{13,17-20}$ introduces an auxiliary quantity called the Kantorovich potential $u(\mathbf{r})$, in which $V_{e e}^{\mathrm{SCE}}[\rho]$ can be obtained according to

$$
\begin{aligned}
V_{e e}^{\mathrm{SCE}}[\rho]=\max _{u} \int u(\mathbf{s}) \rho(\mathbf{s}) \mathrm{d} \mathbf{s}, \\
\text { s.t. } \sum_{i=1}^{N} u\left(\mathbf{r}_{i}\right) \leq \sum_{i=1}^{N} \sum_{j>i}^{N} \frac{1}{\left|\mathbf{r}_{i}-\mathbf{r}_{j}\right|}, \quad \forall\left\{\mathbf{r}_{i}\right\}_{i=1}^{N} .
\end{aligned}
$$

The Kantorovich dual problem (6) is a linear programming problem with respect to $u$, and has the potential of treating general systems with an arbitrary electron density. However, the Kantorovich problem introduces an infinite number of linear constraints due to the arbitrary choice of $\left\{\mathbf{r}_{i}\right\}_{i=1}^{N}$, and cannot be directly implemented in practice. 
Our novel method to overcome the difficulty of infinite number of constraints in the Kantorovich problem reads as follows. First note that the long-range asymptotic behavior of the Kantorovich potential is

$$
u(\mathbf{r})=v(\mathbf{r})+C,
$$

where $\mathrm{C}$ is a constant chosen such that the function $v(\mathbf{r})$ vanishes at infinity and satisfies ${ }^{12,13}$

$$
v(\mathbf{r}) \sim \frac{N-1}{|\mathbf{r}|} \text { as } \quad|\mathbf{r}| \rightarrow \infty .
$$

Without loss of generality we refer to $v(\mathbf{r})$ also as the Kantorovich potential in the following discussion. We introduce a functional $g[v]$ of $v(\mathbf{r})$ by

$$
g[v]=\min _{\left\{\mathbf{r}_{i}\right\}} \sum_{i=1}^{N} \sum_{j>i}^{N} \frac{1}{\left|\mathbf{r}_{i}-\mathbf{r}_{j}\right|}-\sum_{i=1}^{N} v\left(\mathbf{r}_{i}\right),
$$

where the minimization is performed over all possible choices of the positions of the $N$ electrons. The Kantorovich dual problem (6) can then be written as

$$
\begin{gathered}
V_{e e}^{\mathrm{SCE}}[\rho]=\max _{v, C}\left(\int v(\mathbf{s}) \rho(\mathbf{s}) \mathrm{d} \mathbf{s}+N C\right), \\
\text { s.t. } g[v] \geq N C .
\end{gathered}
$$

Here we have used the normalization condition of the electron density, $\int \rho(\mathbf{r}) \mathrm{d} \mathbf{r}=N$. Eq. (10) is a constrained optimization problem with one inequality constraint, which can be solved by defining a Lagrangian

$$
L[v, C, \lambda]=-\int v(\mathbf{s}) \rho(\mathbf{s}) \mathrm{d} \mathbf{s}-N C-\lambda(g[v]-N C) .
$$

The Karush-Kuhn-Tucker (KKT) condition ${ }^{21}$ states that the optimal $\left(v^{*}(\mathbf{r}), C^{*}, \lambda^{*}\right)$ should satisfy the first order necessary condition

$$
\begin{aligned}
\frac{\delta L}{\delta v}\left(v^{*}, C^{*}, \lambda^{*}\right) & =0, \\
\frac{\partial L}{\partial C}\left(v^{*}, C^{*}, \lambda^{*}\right) & =0, \\
g\left[v^{*}\right]-N C^{*} & \geq 0, \\
\lambda^{*} & \geq 0, \\
\lambda^{*}\left(g\left[v^{*}\right]-N C^{*}\right) & =0 .
\end{aligned}
$$

Eq. (12b) implies that

$$
\lambda^{*}=1>0,
$$

which satisfies Eq. (12d). Combining Eq. (13) and (12e) (called the complementary slackness condition), we have

$$
g\left[v^{*}\right]=N C^{*}
$$

Therefore the constrained optimization problem (10) can be solved by eliminating the parameter $C$, resulting in a nested unconstrained optimization problem (for simplicity we drop the stars in the superscripts)

$$
V_{e e}^{\mathrm{SCE}}[\rho]=\max _{v}\left(\int v(\mathbf{s}) \rho(\mathbf{s}) \mathrm{d} \mathbf{s}+g[v]\right) .
$$

We also remark that Eq. (15) can be viewed as a saddle point problem

$$
V_{e e}^{\mathrm{SCE}}[\rho]=\max _{v} \min _{\left\{\mathbf{r}_{i}\right\}} h\left[v,\left\{\mathbf{r}_{i}\right\}\right]
$$

with

$$
\begin{aligned}
& h\left[v,\left\{\mathbf{r}_{i}\right\}\right]= \\
& \quad \int v(\mathbf{s}) \rho(\mathbf{s}) \mathrm{d} \mathbf{s}+\sum_{i=1}^{N} \sum_{j>i}^{N} \frac{1}{\left|\mathbf{r}_{i}-\mathbf{r}_{j}\right|}-\sum_{i=1}^{N} v\left(\mathbf{r}_{i}\right) .
\end{aligned}
$$

The numerical treatment of the saddle point problem (16) is also difficult and is beyond the scope of this paper. Here we solve Eq. (15) via a nested unconstrained optimization approach.

The minimization problem (9) for calculating $g[v]$ poses some hidden difficulties: at any set of minimizers $\left\{\mathbf{r}_{i}\right\}$, it must hold that

$$
-\sum_{j \neq 1}^{N} \frac{\mathbf{r}_{1}-\mathbf{r}_{j}}{\left|\mathbf{r}_{1}-\mathbf{r}_{j}\right|^{3}}-\nabla v\left(\mathbf{r}_{1}\right)=0 .
$$

This is precisely Eq. (7) in Ref. 14. Thus, at the exact dual potential $v(\mathbf{r})$, one recovers the co-motion functions $\mathbf{f}_{i}\left(\mathbf{r}_{1}\right)$ by fixing $\mathbf{r}_{1}$ and minimizing (9) with respect to $\mathbf{r}_{2}, \ldots, \mathbf{r}_{N}$. In particular, since (18) holds for arbitrary $\mathbf{r}_{1}$, the minimizing set $\left\{\mathbf{r}_{i}\right\}$ is not unique. As a consequence, the functional derivative $\frac{\delta g[v]}{\delta v}(\mathbf{r})$ cannot be analytically computed for the exact Kantorovich dual potential $v(\mathbf{r})$. Thus we use derivative-free methods ${ }^{22,23}$ to solve the outer optimization of the Kantorovich dual problem (15). The inner optimization (9) and (18) for calculating $g[v]$ is a standard optimization problem and is solved by the quasi-Newton method. Our numerical results indicate that this hybrid approach can indeed solve atoms and small molecules with reasonable parameterization of the Kantorovich potential.

Special care should be taken when parameterizing $v(\mathbf{r})$ numerically. The long range asymptotic behavior (8) indicates that the size of the computational domain needed to represent $v(\mathbf{r})$ is much larger than the size of the domain to represent the electron density $\rho(\mathbf{r})$. For smooth $v(\mathbf{r})$, it turns out that the correct asymptotic behavior of $v(\mathbf{r})$ can be efficiently preserved by introducing a pseudocharge associated to $v(\mathbf{r})$, denoted by $m(\mathbf{r})$, i.e.,

$$
v(\mathbf{r})=\int \frac{m\left(\mathbf{r}^{\prime}\right)}{\left|\mathbf{r}-\mathbf{r}^{\prime}\right|} \mathrm{d} \mathbf{r}^{\prime} .
$$

The asymptotic behavior (8) translates to the following constraint on $m(\mathbf{r})$ :

$$
\int m(\mathbf{r}) \mathrm{d} \mathbf{r}=N-1
$$


Compared to $v(\mathbf{r})$ which decays as $(N-1) /|\mathbf{r}|$ for large $|\mathbf{r}|$, physical intuition suggests that the support size of $m(\mathbf{r})$ should be much smaller and is comparable to the support size for the electron density $\rho(\mathbf{r})$, as shall be confirmed by our numerical results below. Note that the parametrization in Eq. (19) does not restrict the set of admissible $v(\mathbf{r})$ as long as $v(\mathbf{r})$ is sufficiently smooth, since one can simply define $m(\mathbf{r})$ as $-\Delta v /(4 \pi)$.

For strictly 1D systems however, we remark that the co-motion functions are discontinuous ${ }^{13,15}$. In particular, Eq. (18) implies that $\nabla v(\mathbf{r})$ is discontinuous for these systems, and the pseudocharge will consist of $\delta$-functions and is difficult to discretize. For strictly $1 \mathrm{D}$ systems, we therefore discretize $v(\mathbf{r})$ directly on a grid which matches the asymptotic condition (8).

\section{NUMERICAL RESULTS}

a. Beryllium atom. To illustrate the performance of the nested optimization method in practice, we first study the beryllium atom with 4 electrons. Similar to Ref. 12, the electron density is provided non-selfconsistently by a configuration interaction calculation with Slater-type orbitals ${ }^{24,25}$. Specifically, $\rho(\mathbf{r})$ is a linear combination of terms $r^{j} \mathrm{e}^{-\lambda r}$ with $j=0,1,2$. Since $\rho(\mathbf{r})$ for beryllium is spherically symmetric, the co-motion functions can be obtained semi-analytically ${ }^{12}$ with numerical optimization performed on the angular part of each co-motion function. Our calculation gives $V_{e e}^{\mathrm{SCE}}[\rho]=4 \times 0.812132$.

For the Kantorovich dual formulation, we try to expand the pseudocharge $m(\mathbf{r})$ as a linear combination of Gaussian basis functions. For simplicity, we initially parametrize the pseudocharge $m(\mathbf{r})$ by a single Gaussian function as

$$
m(\mathbf{r} ; \sigma)=\frac{N-1}{\left(2 \pi \sigma^{2}\right)^{3 / 2}} \mathrm{e}^{-\frac{\mathbf{r}^{2}}{2 \sigma^{2}}}
$$

with the value $\sigma$ left to be determined in the optimization procedure. The corresponding Kantorovich potential has the analytic form

$$
v(\mathbf{r} ; \sigma)=\int \frac{m\left(\mathbf{r}^{\prime}\right)}{\left|\mathbf{r}-\mathbf{r}^{\prime}\right|} \mathrm{d} \mathbf{r}^{\prime}=\frac{N-1}{|\mathbf{r}|} \operatorname{erf}\left(\frac{|\mathbf{r}|}{\sqrt{2} \sigma}\right) .
$$

The nested optimization method gives $V_{e e}^{\mathrm{SCE}, 1}[\rho]=4 \times$ 0.647 with $\sigma=0.8630$, and the relative error of $V_{e e}^{\mathrm{SCE}}$ is $20.3 \%$. The result can be significantly improved by parameterizing the pseudocharge $m$ by a sum of two concentric Gaussian functions:

$m(\mathbf{r})=(N-1)\left(\cos ^{2}(\vartheta) \frac{\mathrm{e}^{-\frac{\mathbf{r}^{2}}{2 \sigma_{1}^{2}}}}{\left(2 \pi \sigma_{1}^{2}\right)^{3 / 2}}+\sin ^{2}(\vartheta) \frac{\mathrm{e}^{-\frac{\mathbf{r}^{2}}{2 \sigma_{2}^{2}}}}{\left(2 \pi \sigma_{2}^{2}\right)^{3 / 2}}\right)$,

which yields $V_{e e}^{\mathrm{SCE}, 2}[\rho]=4 \times 0.7995$ with parameters $\sigma_{1}=0.4507, \sigma_{2}=1.862, \vartheta=0.6872$. The relative error of $V_{e e}^{\mathrm{SCE}}[\rho]$ is significantly reduced to $1.6 \%$, which is quite small given that only 3 parameters are employed. The corresponding Kantorovich potential $v(\mathbf{r})$ is shown in Fig. 1, in comparison to the (numerically) exact potential obtained via the co-motion formulation. As for $V_{e e}^{\mathrm{SCE}}$, the potential $v(\mathbf{r})$ with pseudocharge (23) agrees remarkably well with the exact potential. To further improve the result, one can use a larger number of Gaussian basis functions to represent $m(\mathbf{r})$. Note that this Ansatz implicitly assumes that the exact $v(\mathbf{r})$ is smooth, which is indeed the case for this example.

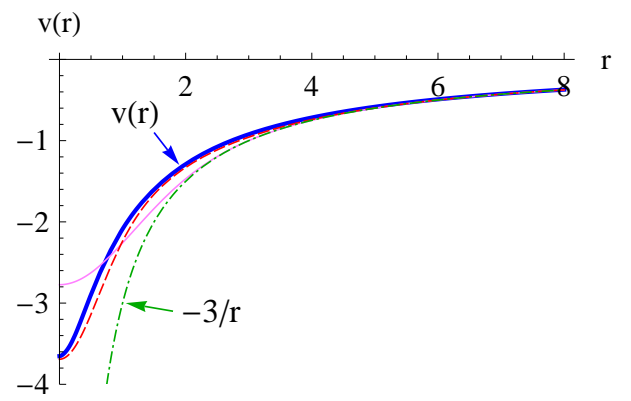

FIG. 1. (Color online) Kantorovich potential $v(\mathbf{r})$ for the beryllium atom: co-motion formulation (thick blue solid line), Kantorovich dual formulation with the pseudocharge $m(\mathbf{r})$ parametrized by a single Gaussian (thin magenta solid line) and by the sum of two Gaussians (red dashed line). The green dot-dashed line shows the asymptotic expansion (8).

b. Quantum wire. Next we study a model quantum wire system in $1 \mathrm{D}$, for which the co-motion formulation can also be solved semi-analytically ${ }^{14}$. The system consists of $N=4$ electrons and the Hamiltonian reads

$$
H=-\frac{1}{2} \sum_{i=1}^{N} \frac{\partial^{2}}{\partial x_{i}^{2}}+\sum_{i=1}^{N} \sum_{j>i}^{N} w_{b}\left(x_{i}-x_{j}\right)+\sum_{i=1}^{N} v_{\mathrm{ext}}\left(x_{i}\right),
$$

where $v_{\text {ext }}(x)=\frac{1}{2} \omega^{2} x^{2}$ is a confining potential and

$$
w_{b}(x)=\frac{\sqrt{\pi}}{2 b} \exp \left(\frac{|x|^{2}}{4 b^{2}}\right) \operatorname{erfc}\left(\frac{|x|}{2 b}\right)
$$

is the effective Coulomb interaction. By increasing the length scale $L \equiv 2 \omega^{-1 / 2}$, the system approaches the SCE limit due to the long-range effective Coulomb interaction $w_{b}(x)$. Concretely, as $L$ increases from 4.5 to 14 , the quantum wire system transforms from a weakly correlated system with 2 peaks in the electron density to a strongly correlated system with 4 peaks in the electron density $^{14}$, which cannot be described by the local density approximation $(\mathrm{LDA})^{3}$ of the KS exchange-correlation functional.

We discretize the Hamiltonian by Hermite functions. The electron density is represented numerically on a grid and is obtained via self-consistent field iterations (SCF). In the Kantorovich dual formulation, we avoid the pseudocharge formulation for this example since the derivative $v^{\prime}(x)$ of the exact dual potential is not continu- 
ous $^{13,15}$ and the pseudocharge consists of $\delta$-functions. Instead, we discretize $v(x)$ directly on a uniform grid. The number of grid points is a compromise between accurate parametrization of $v(x)$ and feasibility of the optimization (15). We focus on the cases $L=6$ and $L=14$, and choose the grid spacing $\Delta x_{L}$ somewhat heuristically as $\Delta x_{6}=\frac{3}{2}$ and $\Delta x_{14}=4$. We allow $v(x)$ at the grid points $-M_{L},-M_{L}+\Delta x_{L}, \ldots, M_{L}$ with $M_{6}=7.5$ and $M_{14}=28$ to be determined by the optimization procedure, and fix $v(x)$ by the asymptotic formula (8) at grid points $|x|>M_{L}$. Between grid points, we use piecewise cubic Hermite interpolation. Additionally, due to the even symmetry $v(x)=v(-x)$ it suffices to optimize $v(x)$ for $x \geq 0$ only. The interval $\left[-M_{L}, M_{L}\right]$ (almost) covers the support of the electron density $\rho(x)$ and corresponds to the characteristic shape of $\rho(x)$, which we try to reproduce by the SCF iteration. As starting point for the SCF iteration, we convolve the exact $v(x)$ and $\rho(x)$ from the co-motion formulation with a Gaussian with variance $\frac{L}{6}$ and $\frac{L}{4}$, respectively. We use linear mixing with parameter $\lambda=0.1$.

Fig. 2 shows the Kantorovich potential $v(x)$ and the density $\rho(x)$ obtained via our method (after 15 and 25 SCF iterations for $L=6$ and $L=14$, respectively), in comparison to the (numerically exact) co-motion formulation. The Kantorovich dual formulation correctly reproduces the strong interaction limit $(L=14)$ with 4 peaks in the electron density. While the results match

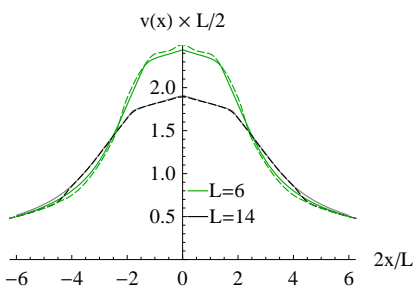

(a)

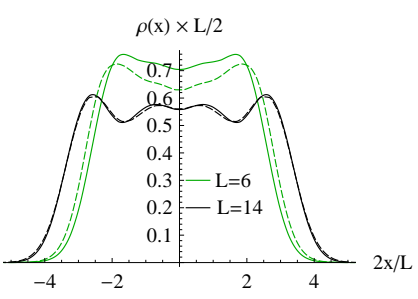

(b)
FIG. 2. (Color online) Comparison of the Kantorovich potential $v(x)$ (a) and density $\rho(x)$ (b) obtained via the "exact" co-motion formulation ${ }^{14}$ (solid lines) and our dual formulation (dashed lines), respectively. The green (upper) curves correspond to $L=6$, and the black (lower) curves correspond to $L=14$.

quite well for $L=14$, one notices a deviation of the density $\rho(x)$ from the co-motion reference for $L=6$. This observation is also reflected by the values of $V_{e e}^{\mathrm{SCE}}$ (after the SCF iteration) shown in Table I. Namely, the relative error of $V_{e e}^{\mathrm{SCE}}$ for $L=14$ is much smaller than for $L=6$. The deviation is likely due to numerical difficulties in the maximization (15). As mentioned above, we make use of the Nelder-Mead simplex algorithm ${ }^{23}$ which is a derivative-free optimization method for the outer optimization. Unfortunately, the results shown in Fig. 2 depend quite sensitively on the parametrization of $v(x)$, e.g., the choices of the above $\Delta x_{L}$ and $M_{L}$. For different choices, $v(x)$ might acquire local maxima during the

\begin{tabular}{r|cc}
$\mathrm{L}$ & 6 & 14 \\
\hline "exact" $V_{e e}^{\mathrm{SCE}}$ & 1.025 & 0.3408 \\
\hline dual-K $V_{e e}^{\mathrm{SEE}}$ & 0.9394 & 0.3381 \\
relative error & $8.4 \%$ & $0.8 \%$
\end{tabular}

TABLE I. $V_{e e}^{\mathrm{SCE}}$ of the model quantum wire system in $1 \mathrm{D}$, for the co-motion formulation (reference) and the Kantorovich dual formulation.

SCF iteration. Thus further improvements of the optimization (15) are required, which we leave as work for the future.

c. Trimer molecule. Finally we apply our method to a model trimer in $3 \mathrm{D}$, for which the optimal transport problem cannot be solved with known techniques using the co-motion formulation. For simplicity, the electron density $\rho(\mathbf{r})$ is given non-self-consistently by a sum of three Gaussian functions centered at the points 1,2,3 in Fig. 3a, respectively. Each Gaussian has variance $\frac{1}{2}$, and each point $1,2,3$ has distance 1 from the origin. The normalization of $\rho(\mathbf{r})$ is fixed by the number of electrons $N=2,3,4,5,6$. An isosurface of the electron density is shown in yellow in Fig. 3a.

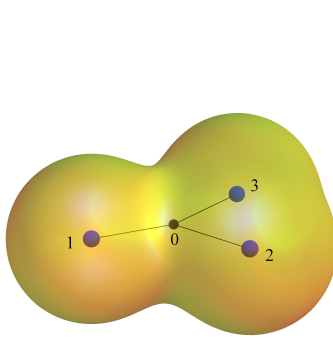

(a)

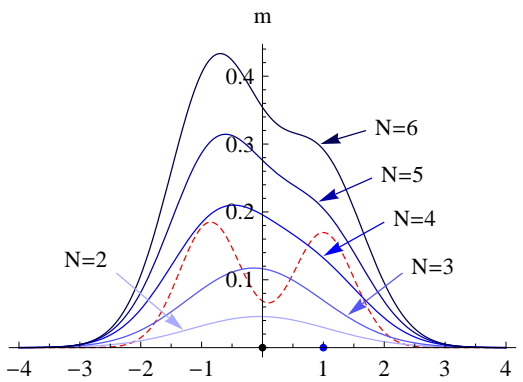

(b)
FIG. 3. (Color online) (a) An isosurface of the electron density $\rho(\mathbf{r})$ (normalized to 1 ) of a model trimer. (b) Optimized pseudocharge $m$ of the trimer molecule (solid blue) and density $\rho(\mathbf{r})$ as is in (a) (dashed red), plotted along the line connecting 1 and 0 in (a). The values 0,1 on the $x$-axis match the corresponding points in (a).

We parametrize the pseudocharge (similar to the density) by a sum of 3 Gaussian functions with the same variance $\sigma$. The centers of the Gaussian functions are located on the black lines in Fig. 3a connecting $0 \rightarrow 1$, $0 \rightarrow 2$ and $0 \rightarrow 3$ respectively, with equal distance $R$ from the origin. The variance $\sigma$ and the distance $R$ are to be determined by the optimization procedure. The results are summarized in Table II, including $V_{e e}^{\mathrm{SCE}}$. Unlike the previous cases for the Be atom and the one dimensional system, to the extent of our knowledge there is no available method that allows us for benchmarking the accuracy of $V_{e e}^{\mathrm{SCE}}$ for the trimer molecule. Nevertheless, the values in Table II are lower bounds on $V_{e e}^{\mathrm{SCE}}$ due to the Ansatz for $m(\mathbf{r})$ in the maximization (15).

Fig. 3b shows the optimized pseudocharge $m(\mathbf{r})$ plot- 


\begin{tabular}{c|ccccc}
$N$ & 2 & 3 & 4 & 5 & 6 \\
\hline$V_{e e}^{\mathrm{SCE}}$ & 0.1973 & 0.4617 & 0.7584 & 1.0711 & 1.3959 \\
$\sigma$ & 1.1073 & 0.9804 & 0.8313 & 0.7741 & 0.7315 \\
$R$ & 0.2260 & 0.5322 & 0.8538 & 0.9062 & 0.9417
\end{tabular}

TABLE II. $V_{e e}^{\mathrm{SCE}}$ of the trimer molecule, and corresponding optimized pseudocharge parameters $\sigma$ and $R$. Due to the specific Ansatz for the pseudocharge (described in the text), the values for $V_{e e}^{\mathrm{SCE}}$ should be regarded as lower bounds.

ted along the line $0 \rightarrow 1$ as in Fig. 3a. Along with increasing $N$, the magnitude of the pseudocharge increases as required by the normalization condition (20). The shape of the pseudocharge develops from a unimodal function for $N=2$ to a bimodal function for $N=6$, indicating growing influence of the distance $R$. For large $N$ the bimodal pseudocharge is biased towards the negative axis around atoms 2 and 3 where the electron density is larger. The increase of $R$ is accompanied by a decrease of $\sigma$, and the support size of the pseudocharge remains approximately the same as $N$ increases, and is comparable to the support size of $\rho(\mathbf{r})$.

\section{CONCLUSION AND FUTURE WORK}

In this paper we present a nested optimization method for solving the Kantorovich dual problem to obtain the exchange correlation functional in the SCE limit for strongly correlated systems. With reasonable parameterization which preserves the asymptotic property of the Kantorovich potential, the Kantorovich dual solution can be obtained for atoms and small molecules. Based on the
Kantorovich dual formulation, one can combine the SCE exchange-correlation functional with existing exchangecorrelation functionals for Kohn-Sham non-interacting kinetic energy in order to improve the performance of KSDFT for strongly correlated systems. For instance, we may mix the SCE exchange-correlation functional with the GGA exchange-correlation functional ${ }^{4-6}$ via linear combination as

$$
E^{\mathrm{SCE}-\mathrm{GGA}}[\rho]=(1-\alpha) E^{\mathrm{SCE}}[\rho]+\alpha E^{\mathrm{GGA}}[\rho],
$$

and obtain $\alpha \in[0,1]$ via a set of benchmark problems.

Due to the difficulty in obtaining the functional derivative $\frac{\delta g[v]}{\delta v}(\mathbf{r})$ by an analytic formula, in practice the outer optimization of the nested optimization method is solved by derivative-free optimization methods. However, our numerical results indicate that the derivative-free methods may get stuck at local minima. Moreover, the derivative-free methods are not suitable for optimizing with respect to a large number of degrees of freedom. More efficient numerical methods need to be developed in order to obtain the Kantorovich dual solution for more general systems in the future.

This work is partially supported by the Technische Universität München (C. M.), and the Laboratory Directed Research and Development Program of Lawrence Berkeley National Laboratory under the U.S. Department of Energy contract number DE-AC02-05CH11231 (L. L.). C. M. thanks the hospitality of the Lawrence Berkeley National Laboratory where the idea of this work starts. We thank Codina Cotar, Gero Friesecke and Brendan Pass for many helpful discussions, as well as Paola Gori-Giorgi for sharing numerical details concerning the quantum wire model.
1 P. Hohenberg and W. Kohn, Phys. Rev. 136, B864 (1964).

${ }^{2}$ W. Kohn and L. J. Sham, Phys. Rev. 140, A1133 (1965).

3 J. P. Perdew and A. Zunger, Phys. Rev. B 23, 5048 (1981).

4 A. D. Becke, Phys. Rev. A 38, 3098 (1988).

${ }^{5}$ C. Lee, W. Yang, and R. G. Parr, Phys. Rev. B 37, 785 (1988).

6 J. P. Perdew, K. Burke, and M. Ernzerhof, Phys. Rev. Lett. 77, 3865 (1996).

7 K. Andersson, B. O. Roos, P. Malmqvist, and P. O. Widmark, Chem. Phys. Lett. 230, 391 (1994).

8 A. I. Liechtenstein, V. I. Anisimov, and J. Zaanen, Phys. Rev. B 52, R5467 (1995).

9 A. J. Cohen, P. Mori-Sánchez, and W. Yang, Chem. Rev. 112, 289 (2012).

10 P. Mori-Sánchez, A. J. Cohen, and W. Yang, Phys. Rev. Lett. 102, 066403 (2009).

11 E. M. Stoudenmire, L. O. Wagner, S. R. White, and K. Burke, Phys. Rev. Lett. 109, 056402 (2012).

12 M. Seidl, P. Gori-Giorgi, and A. Savin, Phys. Rev. A 75, 042511 (2007).

13 G. Buttazzo, L. De Pascale, and P. Gori-Giorgi, Phys. Rev. A 85, 062502 (2012).
${ }^{14}$ F. Malet and P. Gori-Giorgi, Phys. Rev. Lett. 109, 246402 (2012).

15 C. Cotar, G. Friesecke, and C. Klüppelberg, arXiv:1104.0603 (2011).

16 P. Gori-Giorgi, M. Seidl, and G. Vignale, Phys. Rev. Lett. 103, 166402 (2009).

17 L. V. Kantorovich, Dokl. Akad. Nauk. USSR 37, 199 (1942).

18 L. V. Kantorovich, J. Math. Sci. 133, 1381 (2006).

19 C. Villani, Optimal Transport: Old and New (Springer, 2008).

${ }^{20}$ W. Gangbo and A. Święch, Comm. Pure Appl. Math. 51, 23 (1998).

21 J. Nocedal and S. J. Wright, Numerical optimization (Springer Verlag, 1999).

22 R. P. Brent, Algorithms for Minimization Without Derivatives (Dover, 2002).

23 J. C. Lagarias, J. A. Reeds, M. H. Wright, and P. E. Wright, SIAM J. Optim. 9, 112 (1998).

24 G. Friesecke and B. D. Goddard, SIAM J. Math. Anal. 41, 631 (2009).

${ }^{25}$ G. Friesecke and B. Goddard, Multiscale Model. Simul. 7, 
1876 (2009). 